\title{
Recognition masking of auditory duration
}

\author{
HOWARD J. KALLMAN, STEPHEN C. HIRTLE, and DENISE DAVIDSON \\ State University of New York, Albany, New York
}

\begin{abstract}
Forward and backward recognition masking of tonal duration were assessed using young adults as listeners. The task was to label as "short" or "long" a target tone that had a masking tone following or preceding it by a variable silent interstimulus interval (ISI). Forward-and backwardmasking effects were virtually equivalent. On both forward- and backward-masking trials, (1) percentages correct increased with increases in ISIs, (2) the probability of responding "long" to the target increased with increases in ISIs, and (3) the probability of responding "long" increased with increases in the duration of the mask. The equivalence of backward and forward duration masking argues against an interruption theory of duration masking. It is suggested that some form of integration theory, preferably one that includes components unique to the perception of duration, may better account for the results.
\end{abstract}

The ability to identify a target sound is often impaired if a second sound of approximately equal amplitude is presented shortly after the target (see, e.g., Kallman \& Massaro, 1979, 1983; Massaro, 1972, 1975a; Massaro, Cohen, \& Idson, 1976). This effect has been referred to as auditory backward recognition masking (ABRM). Within the 250-msec range of interstimulus intervals (ISIs) between target and mask that results in ABRM, accuracy of target identification generally improves as the ISI increases. This effect holds with pitch, timbre, lateralization, and loudness judgments (Hawkins \& Presson, 1977; Massaro, 1970, 1972, 1975a, 1975b; Massaro et al., 1976; Moore \& Massaro, 1973). To explain these findings, Massaro and his colleagues suggested that as the ISI between target and backward mask increases, more time can be devoted to perceptual processing of the target and, as a result, recognition performance improves (Massaro, 1976; Kallman \& Massaro, 1983; Massaro, 1975a, 1975b).

Massaro and Idson (1976, 1978; Idson \& Massaro, 1977,1980 ) reported a series of experiments that were designed to extend the ABRM findings and accompanying theory to the masking of auditory duration. Such an extension was of particular interest given the prevalent view that the perceptual processing of auditory duration depended on sorts of mechanisms that were different from those used to process other auditory attributes (see Allan, 1979, for a review).

In many of Massaro and Idson's experiments, the listener judged whether a target tone that had a duration of approximately 50 or $90 \mathrm{msec}$ was relatively short or long. In other experiments, the task was to rate the durations of the target on a continuous scale. In most of the studies, the amplitudes of the targets and masks were

The authors thank Shirley Brown for her helpful comments on this article. Requests for reprints should be sent to Howard J. Kallman, Psychology Department, State University of New York at Albany, 1400 Washington Ave., Albany, NY 12222. varied from trial to trial to minimize the use of amplitude cues. Also, the duration of the mask and/or its frequency served as independent variables.

The first result of interest (also reported by Allan \& Rousseau, 1977) was that accuracy of target sound identification improved as the silent ISI between target and backward mask increased from 25 to approximately $200 \mathrm{msec}$. This result mirrored the results of other ABRM experiments and was well described by a quantitative model which assumed that because longer processing times (i.e., longer intertone intervals) allowed for more information to be read out of preperceptual storage, the ability to discriminate between short and long targets increased.

A second result from the identification experiments was that the overall probability of responding "long" to the target increased with increases in the ISI. A parallel result was found in the rating experiments: the rated durations of both the short and long targets increased with increases in the ISI. After offering evidence that this bias to give "long" responses at the longer ISIs was not due simply to decision processes, Massaro and Idson suggested that the perceived duration of a sound increased with increases in the amount of time spent on its processing; Gol'dburt (1961) also reported results consistent with this conclusion. According to Massaro and Idson (1976), "this change in perceived duration occurs simultaneously with changes in discriminability" (p. 347).

A third result found by Massaro and Idson (1976) was that the perceived duration of a target sound appeared to increase with increases in the duration of the mask: in the identification experiments, the probability of a response of "long" increased with increases in the mask duration; in the rating experiments, the average rating of the duration of the target increased with increases in the duration of the mask. Target/mask assimilation effects have also been found in studies of pitch and lateralization masking (Hawkins \& Presson, 1977; Hawkins, Thomas, Presson, Cozic, \& Brookmire, 1974; Kallman 
\& Massaro, 1983; Massaro et al., 1976) and may be due to a modification of the auditory memory of the target sound brought about by the presentation of a mask (Kallman \& Massaro, 1979).

In sum, Massaro and Idson concluded that the backward mask, if presented within $250 \mathrm{msec}$ of the target, interrupts processing of the target. This interruption of processing has the dual effect of decreasing the overall accuracy of judgments of the target's duration and shortening the perceived duration of the target. It is assumed that a secondary effect of the mask is that its characteristics assimilate with the auditory memory of the target.

The conclusion that the mask's effect is due primarily to interruption of target processing may be unwarranted, at least as it pertains to duration masking. One reason for assuming that backward recognition masking is due to interruption is that when pitch, lateralization, or timbre judgments are elicited, backward-masking effects are greater and span a wider range of ISIs than do forward masking effects (Massaro, 1970, 1973; Massaro et al., 1976; Sparks, 1976). If perceptual integration rather than interruption were responsible for recognition masking, one might expect to find roughly equivalent forward- and backward-masking effects (see Massaro, 1970, 1973, 1975b). In contrast to the pitch, lateralization, and loudness results, preliminary research suggests that insofar as the masking of duration is concerned, forward masks may be as effective as backward masks (Kallman \& Morris, 1984). However, the Kallman and Morris (1984) finding of equivalent forward and backward duration masking must be interpreted with caution because some of the significant variables evaluated in the Massaro and Idson experiments, such as mask duration, were not manipulated in the Kallman and Morris study.

The finding of equivalent forward and backward duration-masking effects, if confirmed, would embarrass an interruption-of-processing explanation of backward duration masking. Furthermore, to the extent that forward and backward duration-masking effects are shown to be equivalent, the possibility that forward and backward duration masking are due to the same underlying mechanisms would suggest itself. Since there appear in durationbackward-masking experiments to be three distinct effects attributable to the mask, it is important to determine whether each of these is manifest in a forward-masking situation before concluding that backward and forward masking are due to common mechanisms. The present experiment was designed to provide the necessary contrast between forward and backward duration masking.

\section{METHOD}

\section{Subjects}

Sixteen undergraduates at the State University of New York at Albany served to earn credit in an introductory psychology course. All subjects reported normal hearing. Eight of the subjects were randomly assigned to the forward-mask condition; the other 8 were assigned to the backward-mask condition.

\section{Apparatus and Stimuli}

The subjects were tested individually in a sound-insulated booth. The tonal stimuli were generated by a Coulbourn Instruments Model \$24-05 voltage-controlled oscillator and were gated by a Coulbourn S84-04 rise/fall gate. Both the targets and masks were $700-\mathrm{Hz}$ sinewave tones with rise/fall times of $2 \mathrm{msec}$. The stimuli were amplified by a Coulbourn S82-24 audio mixer-amplifier prior to presentation through Grason-Stadler TDH-50 headphones. Sound levels were calibrated using a Bruel \& Kjaer Model 2203 sound-level meter and Type 4152 artificial ear. A Commodore 64 computer equipped with a Schnedler Systems interface board was used to control the timing of the stimuli, record responses, and present feedback. Subjects responded on a Cardco numeric keypad, and visual feedback was presented over a CRT monitor.

\section{Design and Procedure}

On backward-mask trials, the subject heard the target sound followed by a silent ISI and then the mask. The subject's task was to indicate, by pressing one of two buttons, whether the target was short or long in duration; he/she was instructed to ignore the mask as nearly as possible. The target tone had a full amplitude duration of either 55 or $85 \mathrm{msec}$ and was presented at either 75 or $81 \mathrm{~dB}$ (SPL). The silent ISI that separated the target and mask was 25 , $45,105,165,205,255$, or $500 \mathrm{msec}$; these values were the same as those used by Massaro and Idson (1976), except for the absence, in the present experiment, of a 5-msec ISI and the substitution of a 500-msec ISI for the no-mask condition. The mask had a full amplitude duration of 40,70 , or $100 \mathrm{msec}$ and was presented at either 75 or $81 \mathrm{~dB}$ (SPL). The response interval began at the offset of the target and lasted for $1.5 \mathrm{sec}$. Following the response interval, visual feedback indicating that the target had been short or long was presented for $500 \mathrm{msec}$. Following feedback there was an intertrial interval of $1.5 \mathrm{sec}$ prior to the onset of the next target.

Forward-mask trials were identical to backward-mask trials except that the order of presentation of the mask and target was reversed. On forward-mask trials, the intertrial interval was calculated from the offset of feedback to the onset of the forward mask.

On both backward- and forward-mask trials there were a total of 168 within-subjects experimental conditions [target durations $x$ target amplitudes $\times$ ISIs $\times$ mask durations $\times$ mask amplitudes $(2$ $\times 2 \times 7 \times 3 \times 2$ )]. Within each block of trials, the conditions were presented randomly without replacement. Mask position (forward or backward) was a between-subjects factor.

On Day 1 of the experiment, to introduce the subject to the basic task, he/she was initially presented one block of 168 single-tone trials in which the task was to label each tone as short or long. The procedure and stimulus conditions were identical to those of the masking trials except that no mask was presented. Following the single-tone block, four blocks of 168 masking trials each were presented. A short rest period occurred between blocks. On each of the following 3 days, five blocks of 168 masking trials were presented. Data from the first day were not included in the analysis, and the data from the following 3 days were pooled.

\section{RESULTS}

An analysis of variance (BMDP2V) was performed on the percentages correct, with mask position (forward or backward) serving as a between-subjects factor. The degrees of freedom reported below reflect the Geisser and Greenhouse (1958) correction for violations of sphericity.

The effect of ISIs can be seen in Figure 1. Percentages correct increased with increases in ISIs $[F(2.03,28.40)$ $=40.87, p<.001, M S e=323.19]$; this effect of ISIs 


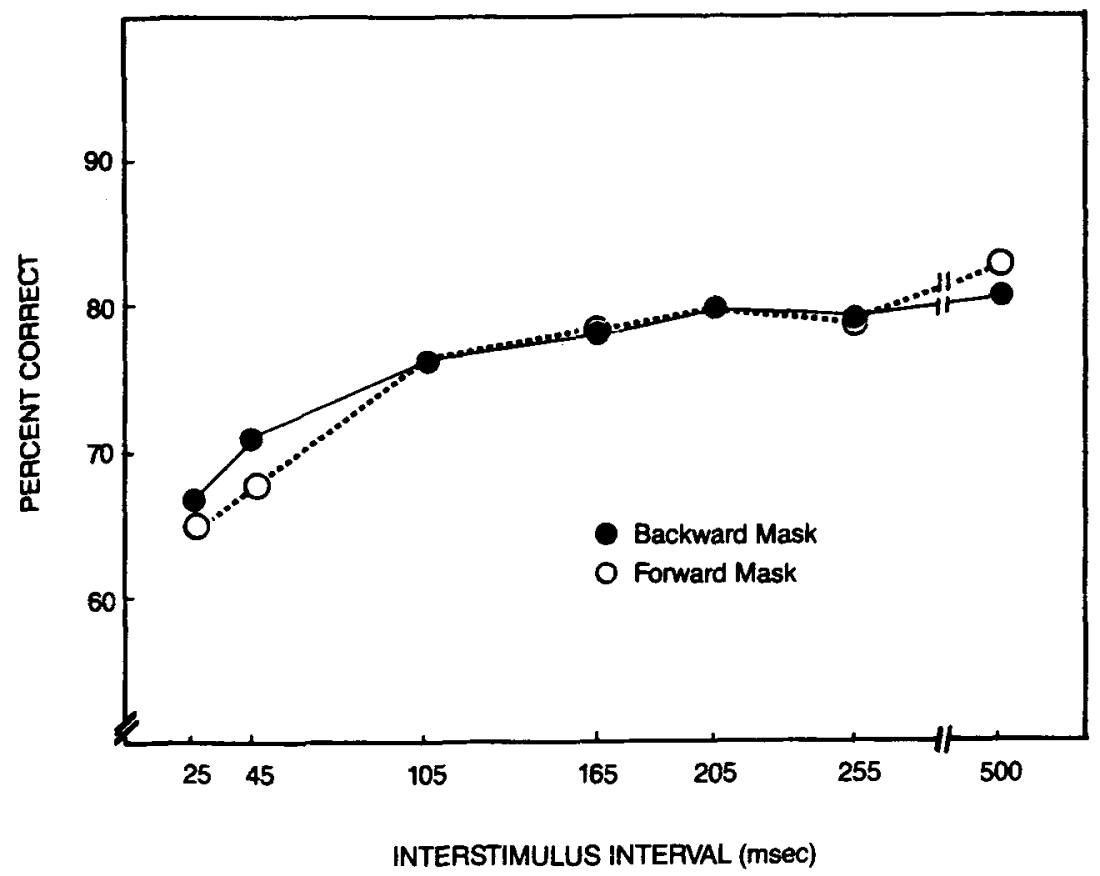

Figure 1. Percentages correct as a function of the interstimulus interval and mask position. In this and all subsequent figures, broken lines connect data points from forward-mask trials and solid lines connect backward-mask data points.

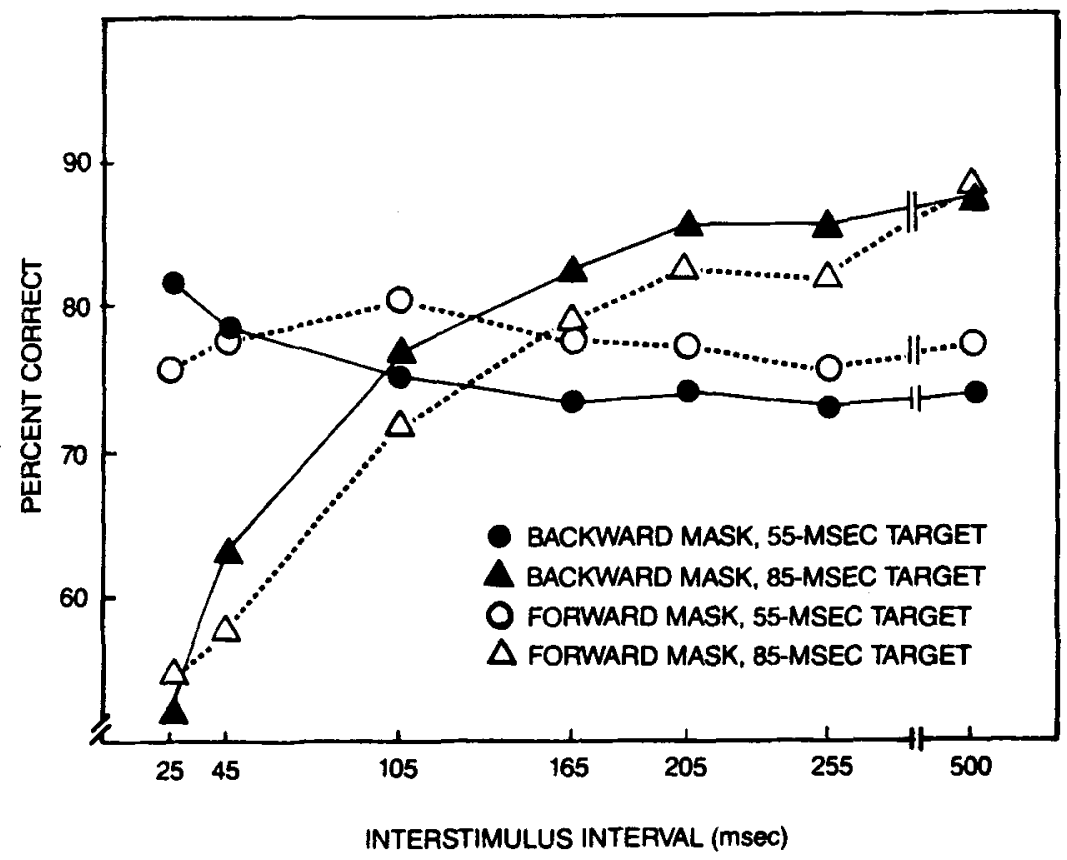

Figure 2. Percentages correct on forward- and backward-masking trials as a function of the interstimulus interval and target duration. 
did not interact with mask position $(F<1, M S e=$ 323.19).

The interaction between target duration and ISIs $[F(1.37,19.18)=12.76, p<.001, M S \mathrm{e}=1,639.72]$ can be seen in Figure 2 . The overall improvement in performance with increases in ISIs was due exclusively to long-target trials; on short-target trials there appears, if anything, to be a slight decrease in accuracy with increases in ISIs. The form of this interaction is consistent with the idea that the perceived duration of a target increases with increases in the ISI. Accordingly, performance improved on long-target trials not only because of the diminished effect of the mask on discriminability as ISI increased, but also because the target, on average, was perceived as longer. On short-target trials, increases in the discriminability of the targets were apparently offset by the perceptual lengthening of the target, which yielded fewer "short" responses (see Massaro \& Idson, 1976). These results of the backward-masking trials essentially repli- cate those of Massaro and Idson. The point of major interest, however, is that the same effects were evident on forward-mask trials.

Average performance at mask durations of 40,70 , and $100 \mathrm{msec}$ was $77.1 \%, 76.4 \%$, and $74.0 \%$ correct, respectively, on backward-mask trials and $76.6 \%, 74.2 \%$, and $75.4 \%$, respectively, on forward-mask trials. The significant effect of mask duration $[F(1.68,23.49)=6.37, p<$ $.01, M S \mathrm{e}=165.66]$ interacted with mask position $[F(1.68,23.49)=4.08, p<.05, M S e=165.66] ;$ higher percentages correct with shorter masks were evident only on backward-mask trials, although this effect was small. Of greater interest is a significant interaction between target duration, mask duration, and interstimulus interval $[F(3.61,50.57)=4.95, p<.01, M S e=243.18]$, which can be seen in Figure 3. This interaction did not differ on forward- and backward-masking trials $[F(3.61,50.67)$ $=1.78, p>.10, M S e=243.18]$. Irrespective of mask position, subjects were most likely, particularly at the

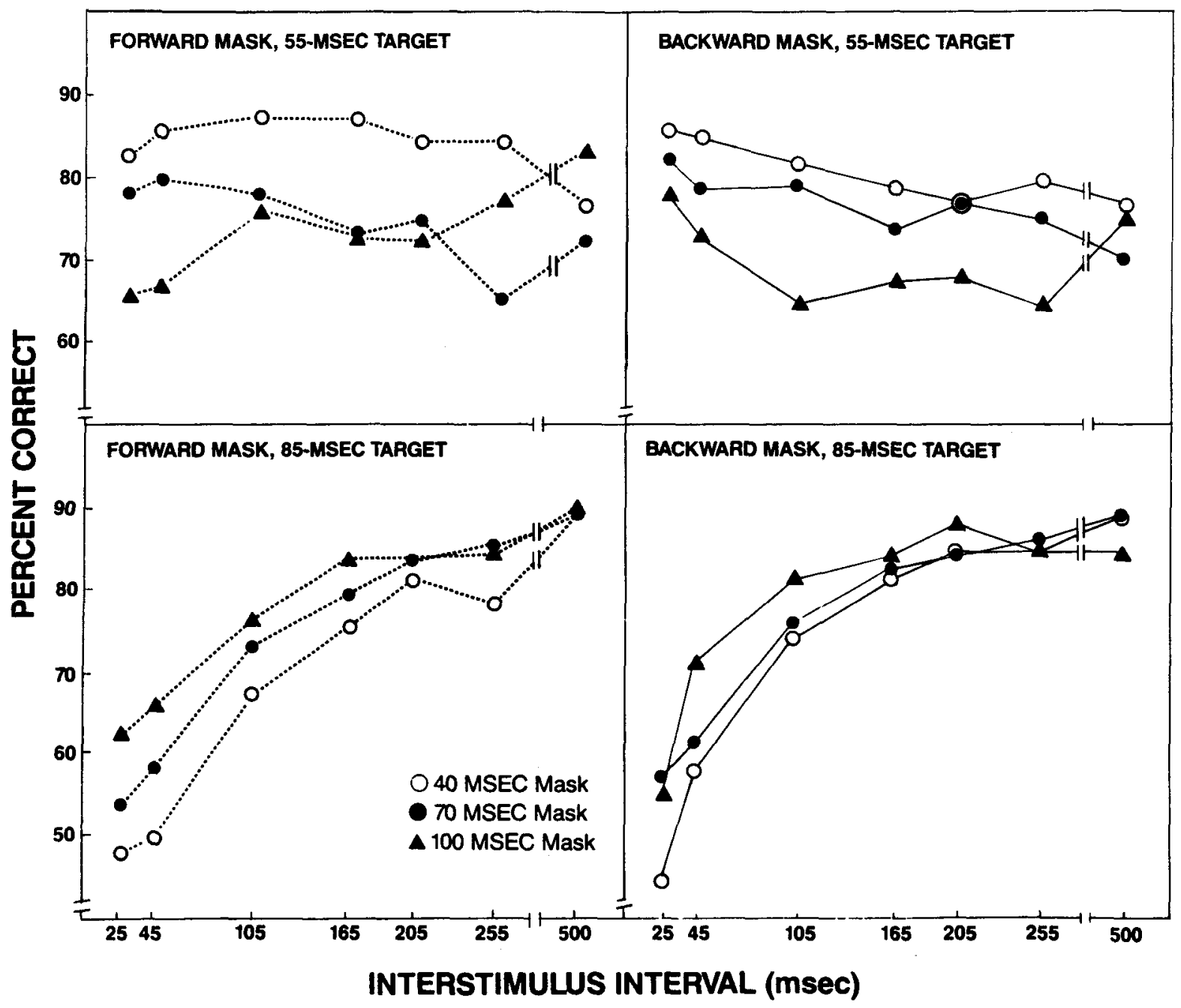

Figure 3. Percentages correct as a function of the interstimulus interval on forward-masking (left panels) and backward-masking (right panels) trials. The upper panels show performance with the 55-msec targets, and the lower panels, performance with the 85msec targets. The curve parameter is the duration of the mask. 
short ISIs, to respond "long" given a long mask. Consequently, on long-target trials, as mask duration increased, there were increases in percentages correct (see the lower panels of Figure 3); on short-target trials, there were corresponding decreases in percentages correct with increases in mask duration (upper panels of Figure 3). The effect of mask duration was significant $[F(1.68,23.49)=6.37$, $p<.01, M S e=165.66]$, and there was a significant interaction with target duration $[F(1.37,19.24)=17.52$, $p<.001, M S e=939.95]$.

On backward-mask trials, mean performance was $77.4 \%$ correct with the $81-\mathrm{dB}$ target and $74.2 \%$ correct with the 75-dB target. On forward-mask trials, percentages correct were 76.7 and 74.1 , respectively. The effect of target amplitude was significant $[F(1,14)=25.63$, $p<.001, M S \mathrm{e}=224.26$ ], although the effect of mask amplitude was not $[F<1, M S e=113.70)$. There was a significant interaction between target and mask amplitude $[F(1,14)=5.08, p<.05, M S e=257.60]$ that did not interact with mask position $(F<1, M S \mathrm{e}=257.60)$, as can be seen in Table 1. Percentages correct were somewhat higher when the target and mask were presented at equal amplitude, although the effect was rather small.

There was a significant interaction between mask amplitude and target duration $[F(1,14)=6.55, p<.05$, $M S e=955.68]$, which did not interact with mask position $(F<1, M S e=955.68$; see Table 2$)$. With an 81$\mathrm{dB}$ mask, percentages correct were higher with the longduration targets; with a 75-dB mask, percentages correct were higher with the short-duration targets. These results suggest that the degree to which the mask "added" to the perceived duration of the target depended on the amplitude of the mask.

The panels of Figures 4 and 5 show the interactions between target amplitude, mask amplitude, target duration, and mask duration, separately for forward- and backwardmask trials. There were two significant higher order interactions involving the target and mask amplitudes. The interaction between target amplitude, mask amplitude, and mask duration was significant $[F(1.72,24.11)=3.92$, $p<.05, M S \mathrm{e}=236.23$ ], as was the interaction between target amplitude, mask amplitude, target duration, and mask duration $[F(1.67,23.35)=17.37, p<.001, M S e$ $=512.86$ ]. These higher order interactions are difficult to interpret; of importance, though, is that neither of these higher order interactions interacted with mask position $(F<1, M S e=236.23$, and $F(1.67,23.35)=3.21$, $p>.10, M S e=512.86]$. Visual inspection of Figures 4 and 5 bears out that the form of these higher order inter-

Table 1

Percentages Correct on Forward- and Backward-Mask Trials as a Function of Target and Mask Amplitudes

\begin{tabular}{lccccc}
\hline & \multicolumn{2}{c}{ Forward Mask } & & \multicolumn{2}{c}{ Backward Mask } \\
\cline { 2 - 3 } \cline { 5 - 6 } & $75 \mathrm{~dB}$ & $81 \mathrm{~dB}$ & & $75 \mathrm{~dB}$ & $81 \mathrm{~dB}$ \\
\hline $75 \mathrm{~dB}$ target & 74.4 & 73.8 & & 75.1 & 73.4 \\
$81 \mathrm{~dB}$ target & 76.1 & 77.3 & 76.4 & 78.5 \\
\hline
\end{tabular}

Table 2

Percentages Correct on Forward- and Backward-Mask Trials as a Function of Target Duration and Mask Amplitude

\begin{tabular}{lccccc}
\hline & \multicolumn{2}{c}{ Forward Mask Trials } & & \multicolumn{2}{c}{ Backward Mask Trials } \\
\cline { 2 - 5 } \cline { 5 - 6 } & $55 \mathrm{msec}$ & $85 \mathrm{msec}$ & & $55 \mathrm{msec}$ & $85 \mathrm{msec}$ \\
\hline $75 \mathrm{~dB}$ mask & 79.2 & 71.4 & 76.4 & 75.0 \\
$81 \mathrm{~dB}$ mask & 75.3 & 75.7 & 74.7 & 77.2 \\
\hline
\end{tabular}

actions was very similar for backward- and forward-mask trials.

\section{GENERAL DISCUSSION}

The results of the present experiment demonstrate that forward and backward masking of auditory duration are comparable. With a forward or a backward mask, (1) discriminability of the short and long targets increased with increases in the ISI separating target and mask, (2) the probability of a "long" response increased with increases in the ISI, and (3) the probability of a "long" response increased with increases in the duration of the mask. The marked similarity between the forward- and backward-masking results suggests that the same mechanisms underlie both types of masking.

An integration theory of duration masking appears attractive in light of the virtual equivalence of backward and forward masking. One possible explanation of these results would assume that duration masking can be explained in terms of perceptual fusion of the target and mask. Such fusion could make it difficult for the subject to "pull apart" the target and mask. As a result, categorization of the target's duration would diminish in accuracy and would reflect the actual durations of both the target and the mask. There are, however, difficulties with any simple integration-based explanation of the present results. In their experiments on backward duration masking, Massaro and Idson (1976; Idson \& Massaro, 1977) used a 5msec ISI as well as the longer ISIs that we used. With the 5-msec ISI, they found that the perceived duration of the target was much greater than would have been predicted from performance at the longer ISIs. This was explained by the assumption that perceptual integration of the target and mask occurred only at very short ISIs (i.e., less than $25 \mathrm{msec}$ ), while interruption occurred at longer ISIs.

Extending the notion of integration to explain the results of both Massaro and Idson's (1976) study and the present study would require the postulation of at least two types of integration. For example, one might assume that integration occurs at more than one level in the nervous system, and that masking at very short ISIs reflects integration near the periphery, whereas masking at the longer ISIs reflects more central integration. Such a distinction between peripheral and central integration was offered by Hawkins and Presson (1977) to explain some of the results of a series of pitch backward-recognition-masking experiments. Parenthetically, Hawkins and Presson's explana- 


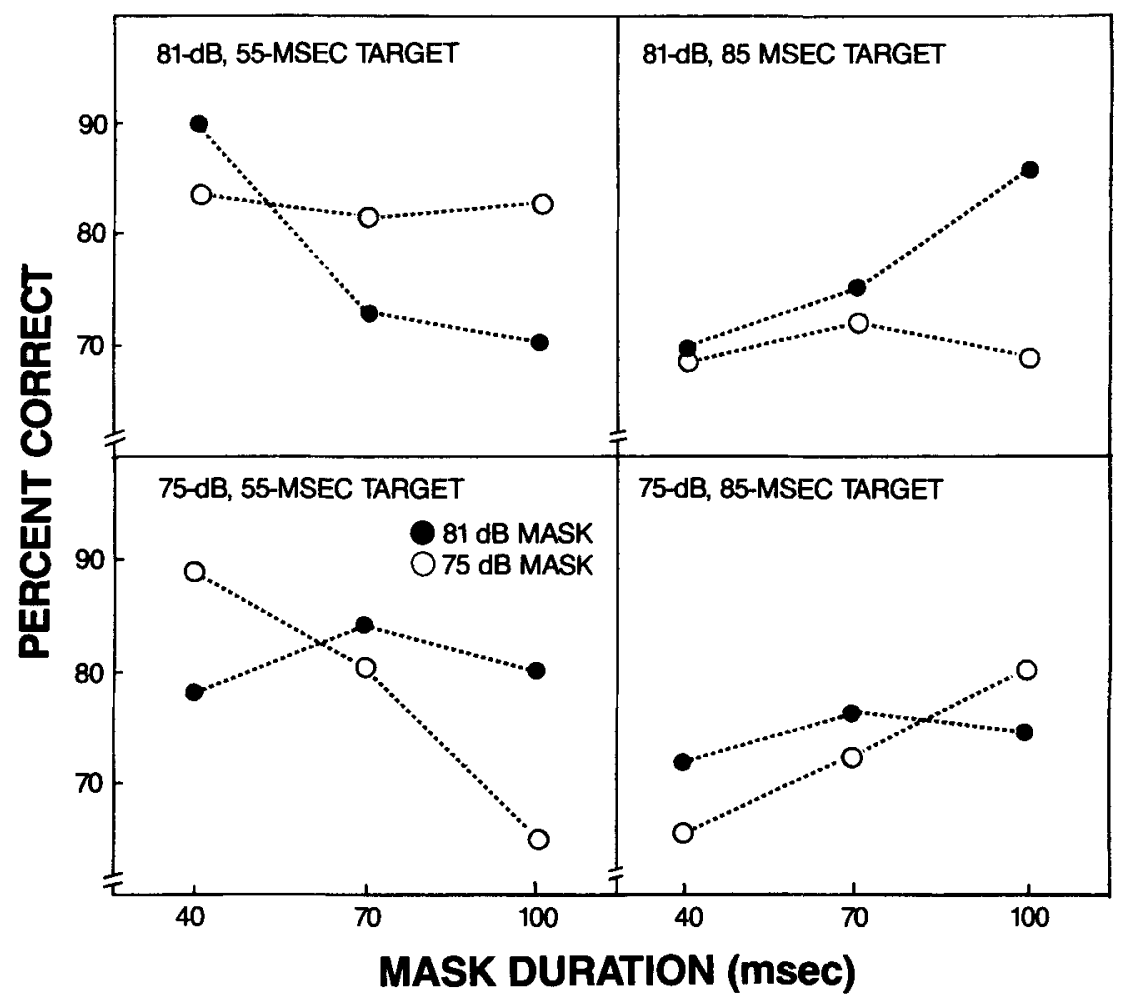

Figure 4. Percentages correct as a function of mask duration on forward-mask trials. Each panel shows performance on one combination of target amplitude and duration. The curve parameter is the amplitude of the mask.

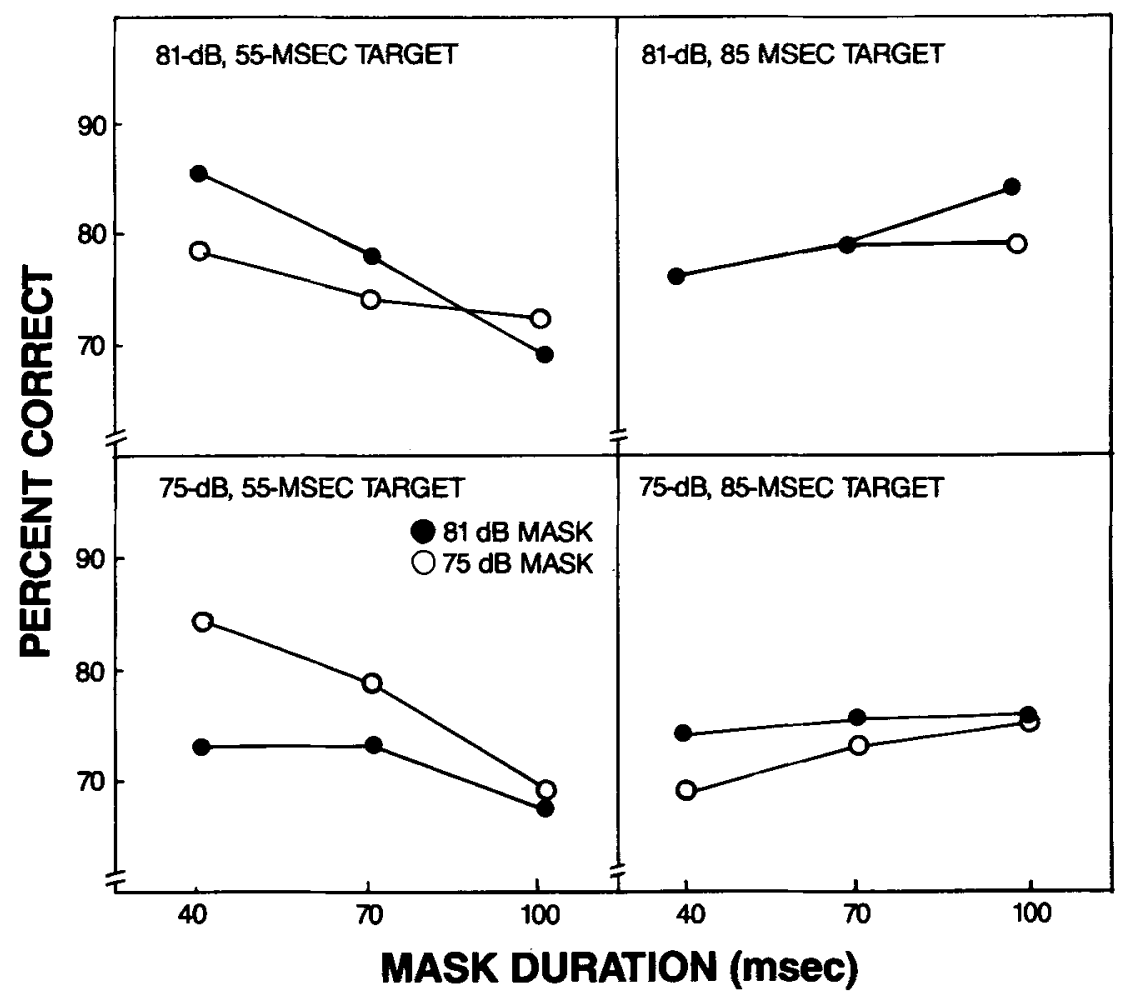

Figure 5. Percentages correct as a function of mask duration on backward-mask trials. Each panel shows performance on one combination of target amplitude and duration. The curve parameter is the amplitude of the mask. 
tion of pitch masking also included an interruption-ofprocessing component. Although the present manipulations do not allow us to separate peripheral and central factors as they affect duration masking, Hawkins and Presson's suggestion that integration effects in backward masking of pitch occur at multiple sites may prove useful to an understanding of duration masking as well.

Although the assumption of perceptual integration could explain some of the present results, there is a logical difficulty that should be noted. If perceptual integration means that the target and mask percepts fuse, we would be hard-pressed to explain why we obtain substantial forward masking in a duration-masking situation but not when other auditory attributes are tested. Integration of the two sounds should affect the processing of a host of attributes, not just duration. Furthermore, phenomenological evidence suggests that, although the mask influences perception of and judgments about the target, the listener has little trouble in hearing that there are two distinct sounds (except possibly at the 5-msec ISI); an integrative process that operated on the sounds as unitary wholes might be expected to result in the perception of a single compound sound. As an alternative, it might be supposed that centrally based integration operates on information about the attributes of sounds rather than on unitary representations of the sounds themselves. In other words, at a central level, it might become difficult to separate out durational information about events that occur close together in time, but this might not apply to as great an extent to other auditory attributes. Given the view that perception of auditory duration depends on unique mechanisms (Allan, 1979), it may be that output from one or more of these mechanisms fails to distinguish clearly between events that occur close together in time.

For example, Thomas and his colleagues (Cantor \& Thomas, 1976; Thomas \& Brown, 1974; Thomas \& Cantor, 1975, 1978; Thomas \& Weaver, 1975) suggested that the perceived duration of a stimulus was a function of both the amount of elapsed time counted from an internal clock and the amount of processing of nontemporal stimulus information that occurs. To the extent that this framework is valid, duration masking could reflect the mask's effect on the output from the internal clock, its effect on the amount of processing of nontemporal stimulus information, or the manner in which these two sources of information are used and combined to arrive at the duration judgment. The equivalence between forward and backward duration masking may be explainable with reference to the sorts of processes proposed by Thomas and Cantor. For example, the fact that there was masking in the backward-masking condition suggests that subjects were generally unable to make a decision about the target until sometime after mask onset. And Massaro and Idson (1976) showed that the duration of the backward mask often affects target duration judgments, even with ISIs of $500 \mathrm{msec}$. This finding suggests that a decision about the duration of the target may not be reached until sometime after information about the duration of the mask has been registered; if this were not the case, there would be no way in which the duration of the mask could influence categorization of the target. Thus, at the time that a decision about the target is made, information about (1) the target's duration, (2) the ISI's duration, and (3) the mask's duration would be registered. Furthermore, in each case, the information could take two forms: information from the internal clock and information about the amount of nontemporal processing. To the extent that any of this information is misassigned to the incorrect stimulus event, masking effects could occur. The probability of misassignment (at least between the target and mask information) could be expected to increase with decreases in the ISI. Furthermore, such misassignment might, in certain instances, be systematic. For example, the increases in perceived duration with increases in ISI could be explained by a tendency to assign clock pulses that occur during a silent interval to one of the contiguous stimulus events. In addition, such factors as a sound's duration and amplitude might influence the amount of time spent on the processing of its nontemporal information and, consequently, influence its perceived duration and the durations of events that occur close in time.

At this stage in our research, it would be premature to offer a detailed theory of duration masking. What is clear is that an interruption theory of the sort proposed by Massaro and Idson (1976) is inadequate to explain the patterns of masking reported presently. Furthermore, duration masking appears to differ in important ways from other sorts of recognition masking and probably reflects mechanisms unique to the perception of duration. Further examination of the interactions between auditory events that occur close together in time should not only serve to contribute to a theory of duration masking but also to a general understanding of how duration is perceived.

\section{REFERENCES}

Allan, L. B. (1979). The perception of time. Perception \& Psychophysics, 26, 340-354.

Allan, L. B., Rousseau, R. (1977). Backward masking in judgments of duration. Perception \& Psychophysics, 21, 482-486.

Cantor, N. E., \& Thomas, E. A. C. (1976). Visual masking effects on duration, size, and form discrimination. Perception \& Psychophysics, 19, 321-327.

GeIsser, S., \& GreEnhouse, S. (1958). An extension of Box's results on the use of the $F$ distribution in multivariate analysis. Annals of Mathematical Statistics, 29, 885-891.

GOL'DBURT, S. N. (1961). Investigations of the stability of auditory processes in micro-intervals of time (new findings in back masking). Biophysics, 6, 809-817.

Hawkins, H. L., Presson, I. C. (1977). Masking and preperceptual selectivity in auditory recognition. In S. Dornic (Ed.), Attention and performance VI (pp. 195-211). Hillsdale, NJ: Erlbaum.

Hawkins, H. L., Thomas, G., Presson, J., Cozic, A., \& Brookmre, D. (1974). Tonal specificity and masking in auditory recognition. Journal of Experimental Psychology, 103, 530-538.

IDSON, W. L., \& MAssaro, D. W. (1977). Perceptual processing and experience of auditory duration. Sensory Processes, 1, 316-337.

IDSON, W. L., \& MAssaro, D. W. (1980). The role of perceived duration in the identification of vowels. Joumal of Phonetics, 8, 407-425. 
Kallman, H. J., Massaro, D. W. (1979). Similarity effects in backward recognition masking. Joumal of Experimental Psychology: Human Perception \& Performance, 5, 110-128.

Kallman, H. J., \& MASSARo, D. W, (1983). Backward masking, the suffix effect, and preperceptual storage. Joumal of Experimental Psychology: Learning, Memory, \& Cognition, 9, 312-327.

Kallman, H. J., \& Morris, M. D. (1984). Duration perception and auditory masking. Annals of the New York Academy of Sciences: Timing and Time Perception, 423, 608-609.

Massaro, D. W. (1970). Preperceptual auditory images. Journal of Experimental Psychology, 85, 411-417.

Massaro, D. W. (1972). Preperceptual images, processing time and perceptual units in auditory perception. Psychological Review, 79, 124-145.

Massaro, D. W. (1973). A comparison of forward versus backward recognition masking. Journal of Experimental Psychology, 100, 434-436.

MASSARO, D. W. (1975a). Backward recognition masking. Joumal of the Acoustical Society of America, 58, 1059-1065.

Massaro, D. W. (1975b). Experimental psychology and information processing. Chicago: Rand McNally.

MAssaro, D. W. (1976). Auditory information processing. In W. K. Estes (Ed.), Handbook of learning and cognitive processes (Vol. 4, pp. 275-320). Hillsdale, NJ: Erlbaum.

Massaro, D. W., Cohen, M. C., Inson, W. L. (1976). Recognition masking of auditory lateralization and pitch judgments. Journal of the Acoustical Society of America, 59, 434-441.
Massaro, D. W., \& IDSON, W. L. (1976). Temporal course of perceived auditory duration. Perception \& Psychophysics, 20, 331-352. Massaro, D. W., \& IDSON, W. L. (1978). Target-mask similarity in backward recognition masking of perceived tone duration. Perception \& Psychophysics, 24, 225-236.

MoORE, J. J., \& MASSARo, D. W. (1973). Attention and processing capacity in auditory recognition. Joumal of Experimental Psychology, 99, 49-54.

SPARKs, D. W. (1976). Temporal recognition masking-or interference? Journal of the Acoustical Society of America, 60, 1347-1353.

Thomas, E. A. C., \& Brown, 1. (1974). Time perception and the filledduration illusion. Perception \& Psychophysics, 16, 449-458.

Thomas, E. A. C., Cantor, N. E. (1975). On the duality of simultaneous time and size perception. Perception \& Psychophysics, 18, 44-48.

Thomas, E. A. C., \& CANToR, N. E. (1978). Interdependence between the processing of temporal and non-temporal information. In J. Requin (Ed.), Attention and performance VII (pp. 43-62). Hillsdale, NJ: Erlbaum.

Thomas, E. A. C., \& Weaver, W. B. (1975). Cognitive processing and time perception. Perception \& Psychophysics, 17, 363-367.

(Manuscript received October 21, 1985; revision accepted for publication May 15, 1986.) 